Indonesian Journal of Biotechnology, December, 2013

Vol. 18, No. 2, pp.75-82

\title{
Stachybotrys chartarum: a novel biological agent for the extracellular synthesis of silver nanoparticles and their antimicrobial activity
}

\author{
Abdel Ghany Tarek Mohamed*
}

Botany and Microbiology Department, Faculty of Science, AL-Azhar University, Cairo, Egypt

\begin{abstract}
Microbial assisted biosynthesis of nanoparticles is a rapidly progressing area of nanobiotechnology. In this paper Stachybotrys chartarum assisted extracellular synthesis of silver nanoparticles (AgNPs) is reported when challenged with $1 \mathrm{mM}$ silver nitrate $\left(\mathrm{AgNO}_{3}\right)$. The characterization of AgNPs was carried out visual observation and UV-Vis spectrophotometry. Further analysis carried out by Fourier Transform Infrared Spectroscopy (FTIR), provides evidence for the presence of proteins as capping agent, which helps in increasing the stability of the synthesized AgNPs. Transmission Electron Microscopy (TEM) investigations confirmed that AgNPs were formed. The synthesized silver nanoparticles were found in the range of 65-108 nm. Finally, the antimicrobial susceptibility of AgNPs synthesized was investigated which exhibited more potent activity against bacteria than fungi compared with using silver nitrate at concentration $1 \mathrm{mM}$.
\end{abstract}

Keywords: Antimicrobial activity, Stachybotrys chartarum, Silver nanoparticles

\section{Introduction}

Nanotechnology can be defined as a research for the design, synthesis, and manipulation of structure of particles with dimension smaller than $100 \mathrm{~nm}$. It has recently become one of the most active research fields in technology and represents an economic alternative for chemical and physical methods of nanopaticles synthesis. These methods of synthesis can be divided on intracellular and extracellular (Ahmad et al., 2005). Nanotechnology is emerging field of science which involves synthesis and development of various nanomaterials. Classically the nanoparticles are produced by physical and chemical methods (Guzman et al., 2012), as these methods are costly, toxic and non eco-friendly. At present, there is a greater need to develop safe, reliable, clean

*Corresponding author:

Abdel Ghany Tarek Muhamed

Botany and Microbiology Department, Faculty of Science, AL-Azhar University, 11884, Cairo, Egypt

E-mail: tabdelghany@yahoo.com,

Phone: 0020105386248 and eco-friendly methods for the preparation of nanoparticles. Biological synthesis process provides a wide range of environmentally acceptable methodology, minimum time required and low cost production. The use of microorganisms in the synthesis of nanoparticles emerges as an eco-friendly and exciting approach. It is well known that many microorganisms like algae, bacteria and fungi produce nanoparticles either intracellularly (Husseiny et al., 2007; Singaravelu et al., 2007; Shiying et al., 2007) or extracellularly (Anilkumar et al., 2007; Riddin et al., 2006). However, the use of eukaryotes, especially fungi, is potentially exciting since they produce large amounts of proteins, thus increasing productivity, and their easy usage in laboratory works is a suitable option in production of metallic nanoparticles among other microorganisms (Sastry et al., 2003). AgNPs are undoubtedly the most widely used nanomaterials among all. Previous studies reported the biosynthesis of AgNPs by different microorganisms such as Fusarium oxysporum (Ahmad et al., 2003), Aspergillus fumigatus (Bhainsa and D'Souza, 
2006), Cladosporium cladosporioides (Balaji et al., 2009), Penicillium sp. (Hemath et al., 2010), Trichoderma harzianum (Prashant and Raja, 2011), Schizophyllum commune (Yen San and Mashitah, 2012) and Penicillium citrinum (Honary et al., 2013). The filamentous fungi possess some advantages over bacteria in nanoparticles synthesis, as most of the fungi are easy to handle, required simple nutrient, possess high wall-binding capacity, as well as intracellular metal uptake capabilities (Sanghi and Verma, 2009).

Microorganisms, such as bacteria and fungi, now play an important role in the remediation of toxic metals through the reduction of the metal ions. The ability of some microorganisms such as bacteria and fungi to control the synthesis of metallic nanoparticles should be employed in the search for new materials (Manda et al., 2006). Different types of nanomaterials like copper, zinc, titanium (Schabes-Retchkiman et al., 2006), magnesium, gold (Gu et al., 2003) and alginate (Ahmad et al., 2006) have been developed, but AgNPs have proved to be most effective as they exhibit potent antimicrobial efficacy against bacteria, viruses and eukaryotic microorganisms. Recent reports indicate that fungi (Kim et al., 2009; Velmurugan et al., 2009), HIV virus (Elechiguerra et al., 2005), bacteriophage viral strain (Narasimha et al., 2012) are susceptible to AgNPs. Duran et al. (2005) reported that AgNPs could have many applications, as in spectrally selected coatings for solar energy absorption, as intercalation material for electrical batteries, as optical receptors, as catalysts in chemical reactions and in bio-labeling. Stachybotrys chartarum is the most commonly found in homes or buildings which have sustained flooding or water damage from broken pipes, roof, wall or floor leaks, condensation. Wet conditions are required to initiate and maintain growth. It is most common on the paper covering of gypsum wall board, but can be found on wallpaper, cellulose based ceiling tiles, paper products, carpets with natural fibers, paper covering on insulated pipes, in insulation material, on wood and wood paneling, and on general organic debris (Cooley et al., 1998; Hodgson et al., 1998). No reports on the synthesis of AgNPs by $S$. chartarum, therefore this study aimed to the biological synthesis of AgNPs using filamentous fungus Stachybotrys chartarum as a novel biological agent, their antibacterial and antifungal activity and characterization of the synthesized AgNPs by UV-Visible spectroscopy and Fourier Transform Infrared Spectroscopy (FTIR) analysis.

\section{Material and Methods \\ Isolation and identification of Stachybotrys chartarum}

S. chartarum was isolated from walls with excessive moisture of building of Faculty of Science, Jazan university, Kingdom of Saudi Arabia (KSA). The fungal isolate was purified by sub-culturing on Czapek-Dox agar medium and was finally maintained on the same slants for identification. The identification of the fungal culture was done on the basis of the microscopic and macroscopic characteristics. The fungus colony of isolate was observed using hand lens and the colony morphology was recorded with respect to color, shape, size and nature of colony. The microscopic characteristics were recorded with using software for image analysis in Faculty of Science, Jazan University, KSA. The cell morphology was recorded with respect to spore chain morphology, hyphae and mycelium structure. The fungus was identified according to Ellis, (1976) and Domsch et al. (1993).

\section{Biosynthesis of silver nanoparticles}

$S$. chartarum was selected for the production of AgNPs. The S. chartarum was inoculated on Czapek-Dox broth medium. The biomass was harvested after complete incubation by filtering through filter paper followed by repeated washing with distilled water for several times to remove any medium component from the biomass. Ten gm (wet weight) of biomass were brought 
in contact with $100 \mathrm{~mL}$ of sterilized double distilled water for $24 \mathrm{~h}$ at $25^{\circ} \mathrm{C}$ in a $250 \mathrm{~mL}$ Erlenmeyer flask and agitated again at 120 rpm. After the incubation, the cell filtrate was obtained by passing it through Whatman filter paper No. 1. The filtrate was treated with aqueous $1 \mathrm{mM} \mathrm{AgNO}$, solution in an Erlenmeyer flask and incubated at room temperature for 4 days. Control (without the silver ion) was also run along with the experimental flask.

\section{UV-visible spectroscopic analysis}

The reduction of silver ions was confirmed by qualitative testing of supernatant by UV-visible spectrophotometer. One $\mathrm{ml}$ of sample supernatant was withdrawn after 24 $\mathrm{hr}$ and absorbance was measured by using UV-visible spectrophotometer between 300$800 \mathrm{~nm}$ at the Regional Center for Mycology and Biotechnology (RCMB) at Al-Azhar University Cairo, Egypt.

\section{Fourier Transform Infrared Spectroscopy and Transmission Electron Microscopy analysis}

The dried powder of AgNPs was subjected to Fourier Transform Infrared Spectroscopy (FTIR) analysis. Two milligrams of the sample was mixed with $200 \mathrm{mg} \mathrm{KBr}$ (FTIR grade) and pressed into a pellet. The sample pellet was placed into the sample holder and FT-IR spectra were recorded in the range $450-4000 \mathrm{~cm}^{-1}$ in FTIR spectroscopy at a resolution of $4 \mathrm{~cm}^{-1}$. Finally, the AgNPs were characterized by Transmission Electron Microscopy (C Joel Jem-1200 EX II. Acc. Voltage $120 \mathrm{KV}$. MAG-medium) at RCMB.

\section{Antimicrobial activity}

The antimicrobial activity of AgNPs was tested against fungi (Aspergillus niger and Candida albicans) and bacteria (Bacillus subtilis and Escherichia coli). Cavities of $6 \mathrm{~mm}$ were made in culture media inoculated with the test organism and were filled with $500 \mu \mathrm{L}$ of AgNPs. After incubation period, the inhibition zone $(\mathrm{mm})$ was recorded. The cavities filled with distilled water served as control. Silver nitrate at $1 \mathrm{mM}$ was used as antimicrobial agent compared with AgNPs. Chemical antibacterial (Ciprofloxacin) and antifungal (Nystatin) agents were used as positive control.

\section{Results and Discussion}

S. chartarum was isolated from walls with excessive moisture covered with susceptible paint. The morphology, color of conidia and other structures examined microscopically help to diagnose the species (Figure 1). As the wet conditions increased the growth of $S$. chartarum increased. This knowledge about $S$. chartarum was confirmed with many authors, where Nelson (2001) reported that S. chartarum has been isolated from gypsum wallboard, pipe insulation, glass fiber wallpaper, and aluminum foil. It typically grows in areas with excessive moisture and temperature variability or on wet material with high cellulose content such as fiberboard, drywall, paper, gypsum board, wood, dust, lint, and wallpaper. Soiled surfaces and surfaces covered with susceptible paint or paper may facilitate $S$. chartarum growth in the absence of dampness (Hossain et al., 2004)

The biological synthesis of AgNPs by different fungi strains was investigated (Duran et al., 2005; Kalimuthu et al., 2008; Honary et al., 2013). S. chartarum was selected for the biosynthesis of AgNPs because of very few or no reports on the synthesis of AgNPs using S. chartarum and their ability to grow on high concentration of silver nitrate $300 \mathrm{ppm}$ (Data not shown). A report by Gurunathan et al. (2009a, b) showed that by controlling the environment

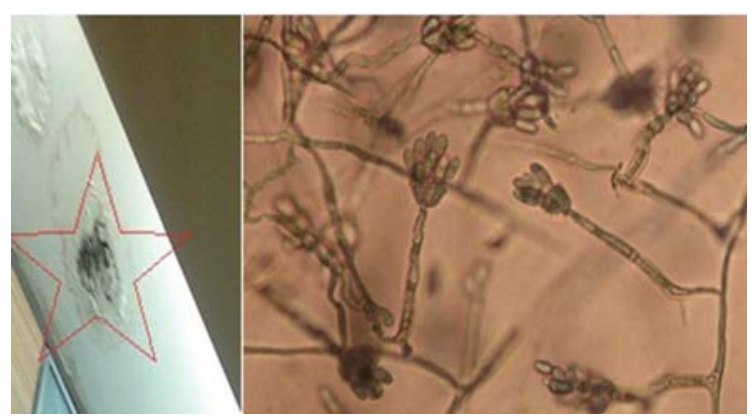

Figure 1. Site of isolation (wet wall) inside star (left) and morphological characterization of $S$. chartarum (right) 


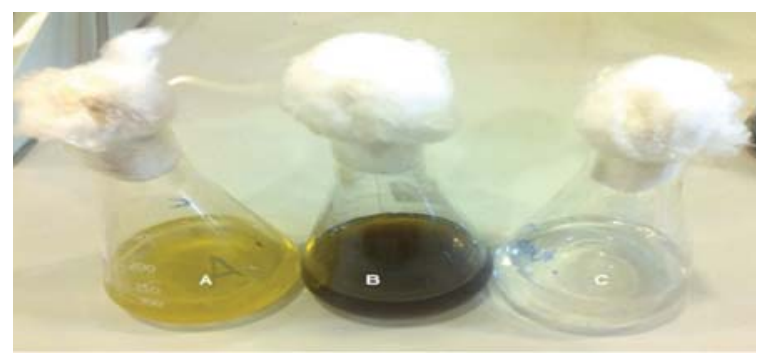

Figure 2. Conversion silver nitrate to nano silver by S. chartarum (A, metabolized medium without silver nitrate inoculated with biomass as a control; B, Distilled water inoculated with biomass and silver nitrate; C, Silver nitrate solution without biomass as a control

of nanoparticle synthesis, AgNPs of various sizes and shapes could be synthesized. Since silver ion is toxic to cells, the concentration of silver ions for the synthesis should be lesser than what we say as the "threshold level", i.e., the conditions beyond which the cells die. So for the synthesis of AgNPs by biomass the optimum concentration that has been patented is $1 \mathrm{mM}$ (Ahamd et al., 2003; Kalimuthu et al., 2008). Silver nitrate $\left(\mathrm{AgNO}_{3}\right)$, upon incubation with the $S$. chartarum biomass, turned dark brown color (Figure 2B), while the positive and negative control flasks remain as such during the $72 \mathrm{~h}$ incubation period (Figures $2 \mathrm{~A}$ and $\mathrm{C}$ ). The generation of dark brown color is due to the surface plasmon resonance exhibited by the nanoparticles. Similar observation was made by several authors (Jaidev and Narasimha 2010; Prashant and Raja, 2011; Yen San and Mashitah, 2012; Honary et al., 2013).
The results of scanning electron microscopy (SEM) showed that AgNPs have a uniform spherical shape, well distributed without aggregation in solution with an average size of about $65-108 \mathrm{~nm}$ (Figure 3). The detected size of AgNPs synthesized by $S$. chartarum may agree or differ with previous scientific papers, this may be due to the species type, incubation period or environmental conditions of microbial growth. Size of AgNPs was 5-25, 10-100, 5-40, 200 and $500 \mathrm{~nm}$ produced by Aspergillus fumigates (Bhainsa and D'Souza, 2006), Cladosporium cladosporioides (Balaji et al., 2009), Trichoderma viride (Fayaz et al., 2010), Geobacter sulfurreducens (Law et al., 2008) and Lactobacillus Strains (Nair and Pradeep, 2002) respectively. Also, from the extracellular secretion of Schizophyllum commune, Lentinus sajar-caju and Pycnoporus sanguineus, it is observed that nanoparticles size was 42.12, 89.76 and $120.6 \mathrm{~nm}$ respectively (Yen San and Mashitah, 2012).

The UV-visible spectrum (Figure 4) showed the peak of AgNPs at $380 \mathrm{~nm}$. It is well known that the size and shape of the AgNPs reflects the absorbance peak. Yen San and Mashitah (2012) reported that broad peak located between wave length 370 and $390 \mathrm{~nm}$ were observed with AgNPs. According to Kannan et al. (2010) wide spectrum range around 390 to $410 \mathrm{~nm}$ was observed. The presence of the broad resonance indicates the agregation of the AgNPs in the solution. Shiv
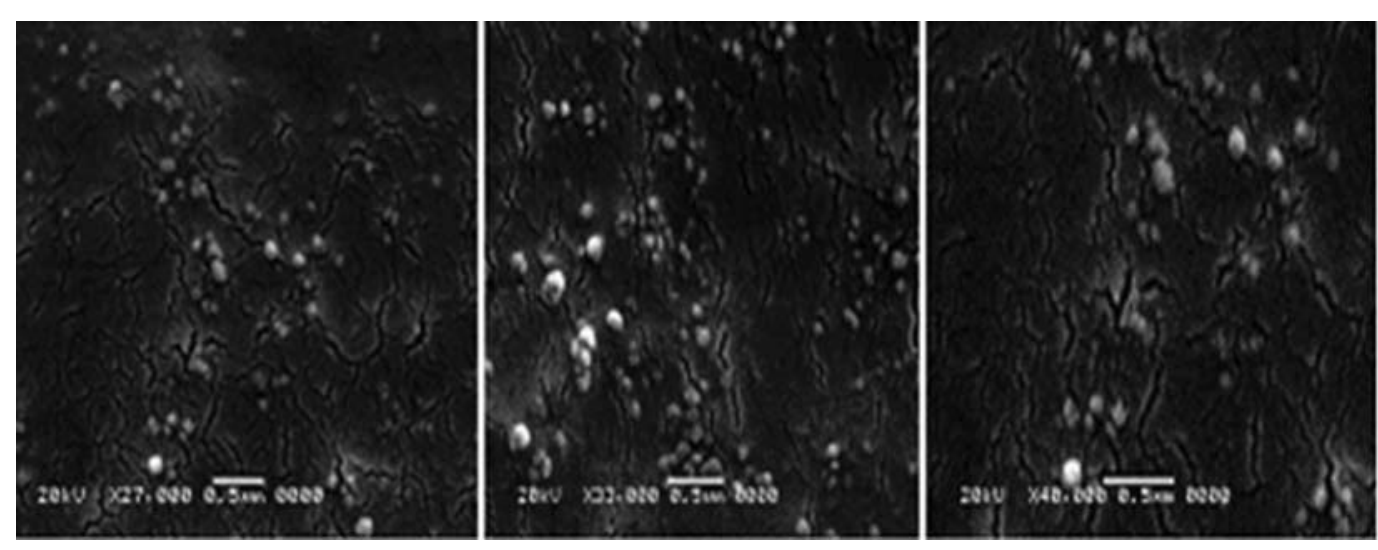

Figure 3. Characterization of AgNPs with different magnification power using Transmission Electron Microscopy 


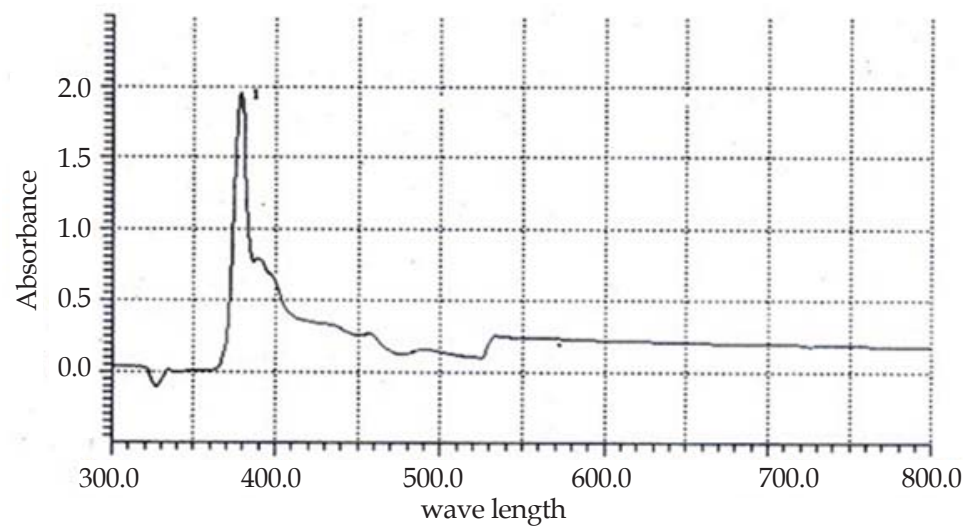

Figure 4. UV-Vis spectrum of silver nanoparticles produced by S. chartarum

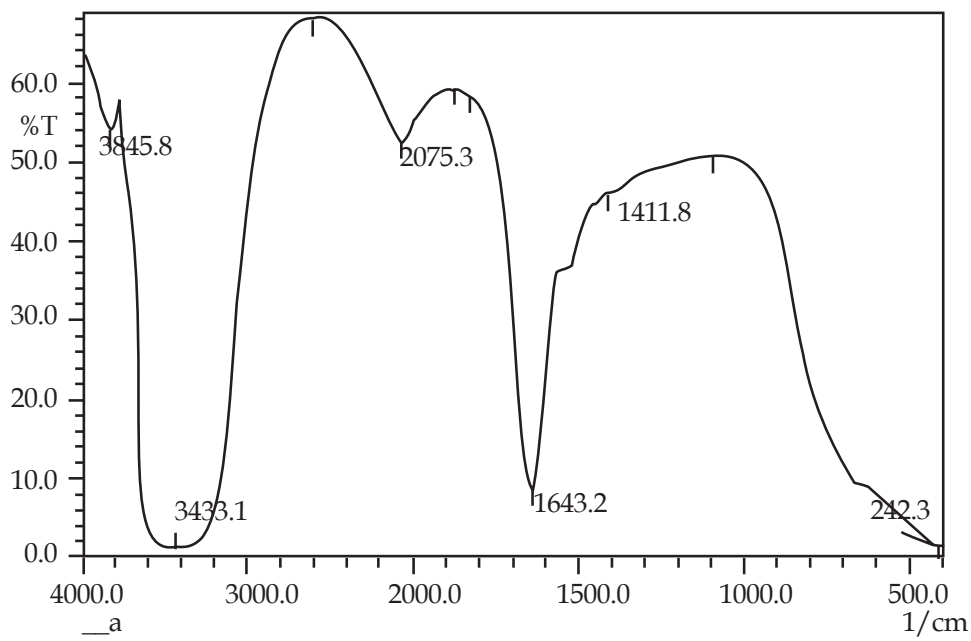

Figure 5. FTIR spectrum of AgNPs formed after $72 \mathrm{~h}$ of incubation of the biomass of $S$. Chartarum

Shankar et al. (2003) suggested that at 370 nm corresponded to the transverse plasmon vibration in AgNPs, whereas the peak at $390 \mathrm{~nm}$ due to excitation of longitudinal plasmon vibrations. FTIR measurements were carried out to identify the possible biomolecules responsible for the reduction of the $\mathrm{Ag}^{+}$ions and capping the bioreduced AgNPs synthesized by fungal biomass. The FTIR spectrum (Figure 5) of the freeze-dried powder of AgNPs revealed the presence of different functional groups. The bands seen at $3433 \mathrm{~cm}^{-1}$ and $2800 \mathrm{~cm}^{-1}$ were assigned to the stretching vibration of primary and secondary amines, respectively. The band existing at $1411 \mathrm{~cm}^{-1}$ can be assigned to the C-N stretching vibrations of aromatic amines.
The band seen at $1643 \mathrm{~cm}^{-1}$ was assigned to the stretching vibration of $\mathrm{C}=\mathrm{C}$. The peak at $1636.17 \mathrm{~cm}^{-1}$ is due to the carbonyl stretch vibrations in the amide linkages of proteins (Basavaraja et al., 2008). These groups may be between amino acid residues in protein and synthesized AgNPs. FTIR spectrum supports the presence of proteins in the synthesis of AgNPs.

Finally, the antimicrobial susceptibility of AgNPs synthesized was investigated (Table 1). AgNPs exhibited more potent activity against bacteria than fungi. However, this compound exhibited no activity on Aspergillus niger. Nanda et al. (2009) has shown that AgNPs are an effective antimicrobial agent against various pathogenic microorganisms. 
Table 1. Antimicrobial activity of silver nanoparticles

\begin{tabular}{lcccc}
\hline \multirow{2}{*}{ Treatments } & \multicolumn{3}{c}{ Antimicrobial activty (inhibition zone mm) against } \\
\cline { 2 - 5 } & \multicolumn{3}{c}{ Bacteria } & Fungi \\
\cline { 2 - 5 } & Bacillus subtilis & Escherichia coli & Candida albicans & Aspergillus niger \\
\hline Control & 0.00 & 0.00 & 0.00 & 0.00 \\
AgNPs $(500 \mu \mathrm{L})$ & 20.2 & 17.0 & 15.0 & 0.00 \\
Silver nitrate $(1 \mathrm{mM})$ & 11.0 & 10.0 & 0.90 & 0.00 \\
Ciprofloxacin & 26.0 & 22.0 & 0.00 & 0.00 \\
Nystatin & 0.00 & 0.00 & 23.0 & 13.0 \\
\hline
\end{tabular}

Ciprofloxacin and Nystatin used as positive control for antibacterial and antifungal respectively

According to Nithya and Ragunathan (2009), the nanosilver was found to have wider antimicrobial activity in Gram negative organisms than the Gram positive one. Recently, according to Arun et al. (2013), AgNPs showed activity against Corynebacterium diphtheriae, Escherichia coli, Bacillus sp., and Staphylococcus aureus and no activity was shown against Pseudomonas aeruginosa. Guzman et al. (2012) stated that the AgNPs may attach to the surface of the cell membrane and disturb its power function of microbe such as permeability and respiration.

\section{Conclusion}

It has been demonstrated that the fungus S. chartarum is capable of producing AgNPs extracellularly. AgNPs showed antibacterial and antifungal activity. Future studies can be conducted to explore applications of the AgNPs generated from the S. chartarum to control of their growth and mycotoxins.

\section{Acknowledgement}

I wish to thank Dr. Mahmoud Al-Asar, researcher in Regional Center of Mycology and Biotechnology, Al- Azhar University, Cairo, Egypt for providing laboratory facilities.

\section{References}

Ahmad, A., Mukherjee, P., Senapati, S., Mandal, D., Khan, M.I., Kumar, R., 2003. Extracellular biosynthesis of silver nanoparticles using the fungus Fusarium oxysporum. Colloids Surf B., 28, 313-318.
Ahmad, Z., Pandey, R., Sharma, S., Khuller, G.K., 2006. Alginate nanoparticles as antituberculosis drug carriers: formulation development, pharmacokinetics and therapeutic potential. Indian journal of chest diseases and allied sciences, 48, (3), 171-176.

Anilkumar, S., Abyaneh, M.K., Gosavi, S.W., Kulkarni, S.K., Pasricha, R., Ahmad, A., Khan, M.I., 2007. Nitrate reductase mediated synthesis of silver nanoparticles from $\mathrm{AgNO}_{3}$. Biotechnol. Lett., 29, 439445.

ArunP.,Shanmugaraju, V., Renga Ramanujam, J., Senthil Prabhu, S., Kumaran, E., 2013. Biosynthesis of Silver Nanoparticles from Corynebacterium sp. and its antimicrobial activity. Int.J.Curr. Microbiol. App.Sci., 2, (3), 57-64.

Balaji, D.S., Basavaraja, S., Bedre Mahesh, D., Prabhakar, B.K., Venkataraman, A., 2009. Extracellular biosynthesis of functionalized silver nanoparticles by strains of Cladosporium cladosporioides. Colloids Surf B, 68, 88-92.

Basavaraja, S., Balaji, S.D., Lagashetty, A., Rajasab, A.H., Venkataraman, A., 2008. Extracellular biosynthesis of silver nanoparticles using the fungus Fusarium semitectum. Materials Res. Bulletin, 43, (5), 1164-1170.

Bhainsa, K.C., D'Souza, S.F., 2006. Extracellular biosynthesis of silver nanoparticles using the fungus Aspergillus fumigatus. Colloids Surf B, 47,160-164.

Cooley, J.D., Wong, W.C., Jumper, C.A., Straus, D.C., 1998. Correlation between 
the prevalence of certain fungi and sick building syndrome. Occup Environ Med., 55, 579-584.

Domsch, K.H, Gams, W., Anderson, T.H., 1993. Compendium of Soil Fungi. V I. IHWVerlag, Eching.

Duran, N., Marcato, P.D., Alves, O.L., Souzaand, G., Esposito, E., 2005. Mechanistic aspects of biosynthesis of silver nanoparticles by several Fusarium oxysporum strains. J. of Nanobiotechnol, 3, (8), 3-8.

Elechiguerra, J. L., Burt, J. L., Morones, J. R., Camacho-Bragado, A., Gao, X., Lara, H. H. , Yacaman, M.J., 2005. Interaction of silver nanoparticles with HIV-1. J. Nanobiotechnol, 3, (6), 1477-3155.

Ellis, M.B. (1976) More Dematiaceous Hyphomycetes. Commonwealth Mycological Institute, Kew, UK.

Fayaz, M., Tiwary, C.S., Kalaichelvan, P.T., Venkatesan, R., 2010. Blue orange light emission frombiogenic synthesized silver nanoparticles using Trichoderma viride. Colloids Surf B, 75, (1),175-178.

Gu, H., Ho, P.L., Tong, E., Wang, L. , Xu, B., 2003. Presenting vancomycin on nanoparticles to enhance antimicrobial activities. Nano lett, 3, (9), 1261-1263.

Gurunathan, S., Kalishwaralal, K., Vaidyanathan, R., Venkataraman, D., Pandian,S.,R.,K., Muniyandi, J., Hariharan, N., Eom, S.H., 2009a. Biosynthesis, purification and characterization of silver nanoparticles using Escherichia coli. Colloids Surf B, 74, (1), 328-335.

Gurunathan, S., Lee, K.,J., Kalishwaralal, K., Sheikpranbabu, S., Vaidyanathan, R., Eom, S.H., 2009b. Antiangiogenic properties of silver nanoparticles. Biomaterials, 30, 6341-6350.

Guzman, M., Dille, J. Godet, S., 2012. Synthesis and antibacterial activity of silver nanoparticles against gram-positive and gram-negative bacteria. Nanomed. Nanotechnol. Biol. and Med, 8, (1), 37-45.

Hemath, N.K., Gaurav, K., Karthik, L., Bhaskara Rao K.V., 2010. Extracellular biosynthesis of silver nanoparticles using the filamentous fungus Penicillium sp. Archives of Applied Science Research, 2, (6), 161-167.

Hodgson, M.J., Morey, P., Leung, W., Morrow, L., Miller, D., Jarvis, B.B., Robbins, H., Halsey, J.F., Storey, E., 1998. Buildingassociated pulomonary disease from exposure to Stachybotrys chartarum and Aspergillus versicolor. J. Occup. Environ. Med., 40, 241-249.

Honary, S., Hamed, B., Eshrat, G., Farzaneh, N., 2013. Green Synthesis of Silver Nanoparticles Induced by the Fungus Penicillium citrinum. Tropical Journal of Pharmaceutical Research, 12, (1), 7-11.

Hossain, M.A., Ahmed, M.,S., Ghannoum, M.,A., 2004. Attributes of Stachybotrys chartarum and its association with human disease. J. Allergy Clin Immunol., 113, 200-208.

Husseiny, M.,I., Ei-Aziz, M.,A., Badr, Y., Mahmoud, M.A. 2007. Biosynthesis of gold nanoparticles using Pseudomonas aeruginosa. Spectrochim Acta [A], 67, 10031006.

Jaidev, L.R., and Narasimha, G., 2010. Fungal mediated biosynthesis of silver nanoparticles, characterization and antimicrobial activity. Colloids and Surf. B: Biointerfaces, 81, 430-433.

Kalimuthu, K., Babu, R.S., Venkataraman, D., Mohd, B., Gurunathan, S., 2008. Biosynthesis of silver nanocrystals by Bacillus licheniformis. Colloids Surf B, 65,150-153.

Kannan, N., Subbalaxmi, S., Ramachandra, M., 2010. Microbial production of silver nanoparticles. Digest J. Nanomaterials and Biostructures , 5, (1), 135-140.

Kim, K.J., Sung, W.S., Suh, B.K., Moon, S.K., Choi, J.S., Kim, J.G., Lee, D.G. 2009. Antifungal activity and mode of action of silver nano-particles on Candida albicans. Biometals, 22, (2), 235-242.

Law, N., Ansari, S., Livens, F.R., Renshaw, J.C., Lloyd, J.R., 2008. The formation of nano-scale elemental silver particles via enzymatic reduction by Geobacter 
sulfurreducens. Appl. Environ. Microbiol., 74, 7090-7093.

Mandal, D., Bolander, M.E., Mukhopadhyay, Sarkar, G., Mukherjee, P., 2006. The use of microorganisms for the formation of metal nanoparticles and their application. J. Appl Microbiol Biotechnol., 69, 485-492.

Nair, B., and Pradeep, T., 2002. Coalescence of nanoclusters and formation of submicron crystallites assisted by Lactobacillus strains. Cryst. Growth Des., 2, 293-298.

Nanda, A., Saravanan, M., 2009. Biosynthesis of silver nanoparticles from Staphylococcus aureus and its antimicrobial activity against MRSA and MRSE. Nanomed: NBM, 5, 452-456.

Narasimha, G., Habeeb, K., Mohammed, A., 2012. Antiviral properties of silver nanoparticles synthesized by Aspergillus sp. Der Pharmacia Lett, 4, (2), 649-651.

Nelson, B.D., 2001. Stachybotrys chartarum: The toxic indoor mold. APBSnet feature story. Internet address: http:/ / www.apsnet. org/online/feature/stachybotrys/. Last accessed on March 29, 2002.

Nithya, R., and Ragunathan, R., 2009. Synthesis of silver nanoparticle using Pleurotus sajor caju and its antimicrobial study. Dig. J. of Nanomaterials and Biostructures, 4, (4), 623- 629.

Prashant, S., and Raja, R.B., 2011. Biological synthesis and characterization of silver nanoparticles using the fungus Trichoderma harzianum. Asian J. Exp. Biol. Sci., 2, (4), 600-605.

Riddin, T., Gericke, M., Whiteley, C., 2006. Analysis of inter and extracellular formation of platinum nanoparticles by Fusarium oxysporum f. sp. lycopersici using response surface methodology. Nanotechnology, 17, 3482-3489.

Sanghi, R., and Verma, P., 2009. Biomimetic synthesis and characterisation of protein capped silver nanoparticles. Bioresour. Technol., 100, (1), 501-504.

Sastry, M.M., Ahmadand, A., khan, I., 2003. Biosynthesis of metal nanoparticles using fungi and Actinomycete. Curr. Sci., 85, 202-206.

Schabes-Retchkiman, P.S., Canizal, G., Herrera-Becerra, R., Zorrilla, C., Liu, H.B., Ascencio, J.A., 2006. Biosynthesis and characterization of $\mathrm{Ti} / \mathrm{Ni}$ bimetallic nanoparticles. Optical materials, 29, (1), 95-99.

Shiv Shankar, S., Ahmad, A., Sastry, M., 2003. Geranium leaf assisted biosynthesis of silver nanoparticles. Biotechnol. Prog., 19, 1627-1631.

Shiying, H., Zhirui, G., Zhang, Y., Zhang, S., Wang, J., Gu, N., 2007. Biosynthesis of gold nanoparticles using the bacteria Rhodopseudomonas capsulata. Mater Lett., 61, 3984-3987.

Singaravelu, G., Arockiamary, J.S., Kumar, V.G., Govindaraju, K., 2007. A novel extracellular synthesis of monodisperse gold nanoparticles using marine alga, Sargassum wightii Greville. Colloids Surf B Biointerfaces, 57, 97-101.

Velmurugan, N., Kumar, G.G., Han, S.S., Nahm, S.K., Lee, S.Y., 2009. Synthesis and characterization of potential fungicidal silver nano-sized particles and chitosan membrane containing silver particles. Iranian polymer J., 18, 383-392.

Yen San, C., and Mashitah, M., 2012. Instantaneous biosynthesis of silver nanoparticles by selected macro fungi. Australian J. Basic and Applied Sciences, 6, (1), 222-226. 